\title{
Stabilization of Mechanical Systems Using Controlled Lagrangians
}

\author{
Anthony M. Bloch ${ }^{1}$ \\ Department of Mathematics \\ University of Michigan \\ Ann Arbor, MI 48109 \\ abloch@math.lsa.umich.edu
}

\author{
Naomi Ehrich Leonard ${ }^{2}$ \\ Dept. of Mechanical and Aerospace Engineering \\ Princeton University \\ Princeton, NJ 08544 \\ naomi@princeton.edu
}

\author{
Jerrold E. Marsden ${ }^{3}$ \\ Control and Dynamical Systems \\ California Institute of Technology 116-81 \\ Pasadena, CA 91125 \\ marsden@cds.caltech.edu
}

\begin{abstract}
We propose an algorithmic approach to stabilization of Lagrangian systems. The first step involves making admissible modifications to the Lagrangian for the uncontrolled system, thereby constructing what we call the controlled Lagrangian. The Euler-Lagrange equations derived from the controlled Lagrangian describe the closed-loop system where new terms are identified with control forces. Since the controlled system is Lagrangian by construction, energy methods can be used to find control gains that yield closed-loop stability. The procedure is demonstrated for the problem of stabilization of an inverted pendulum on a cart and for the problem of stabilization of rotation of a rigid spacecraft about its unstable intermediate axis using a single internal rotor. Similar results hold for the dynamics of an underwater vehicle.
\end{abstract}

\section{Introduction}

In this paper we describe an algorithmic approach to the derivation of stabilizing control laws for Lagrangian systems. The guiding principle is to consider a class of control laws that yield closed-loop dynamics that remain Lagrangian. The advantage of requiring Lagrangian closed-loop dynamics is that stabilization can be understood in terms of energetics. In particular, we can make use of energy methods which automati-

\footnotetext{
${ }^{1}$ Research partially supported by the National Science Foundation PYI grant DMS-91-57556, AFOSR grant F49620-96-10100, a Guggenheim Fellowship and the Inst. for Advanced Study.

${ }^{2}$ Research partially supported by the National Science Foundation under grant BES-9502477 and by the Office of Naval Research under grant N00014-96-1-0052.

${ }^{3}$ Research partially supported by the National Science Foundation under Grant DMS-9302992 and the Department of Energy under Contract DE-FG0395-ER25251.
}

cally provide a Lyapunov function for choosing control gains and proving closed-loop stability. Further, even though work is done by the control forces; there is an extension of the mechanical energy of the system that is conserved; one can think of it as a combined energy available to the mechanism and the control forces. This guarantees, for example, that the control inputs will never need to become very large to achieve stabilization.

Closed-loop dynamics are guaranteed to be Lagrangian by first choosing the closed-loop Lagrangian from a class of controlled Lagrangians we shall elucidate. The controlled Lagrangian then provides the control law: the closed-loop dynamics are the Euler-Lagrange equations derived from the controlled Lagrangian and the new terms that appear in the dynamic equations are identified with the control forces. Rules are imposed in choosing the controlled Lagrangian so that new terms appear only in desired control directions.

The approach is motivated by a result in Bloch, $\mathrm{Kr}$ ishnaprasad, Marsden and Sánchez de Alvarez [1992] for stabilization of unstable middle axis rotation of a rigid spacecraft using a single internal rotor. There, the framework was Hamiltonian and it was shown that the chosen rotor control law was such that the closedloop system was still Hamiltonian. The new Hamiltonian was a modification of the kinetic energy (Hamiltonian) of the uncontrolled spacecraft. The energyCasimir method was used to choose the control gain and thereby guarantee closed-loop stability.

The objective of this paper is to demonstrate how the approach of Bloch, Krishnaprasad, Marsden and Sánchez de Alvarez [1992] can be generalized and made algorithmic. We switch to a Lagrangian framework from a Hamiltonian framework which helps us to systematize the modification of the uncontrolled Lagrangian to get our controlled Lagrangian. The ba- 
sic idea behind our approach was introduced in Bloch, Marsden and Sánchez de Alvarez [1997].

We confine ourselves, in this paper, to controlled Lagrangians that only involve modifications to the kinetic energy of the system. One can also consider modifications to the potential energy for stabilization and tracking purposes. In future work, we intend to incorporate, into our algorithm, modifications to the potential energy such as the addition of symmetry-breaking potentials along the lines of Leonard [1997b]. Other relevant work involving energy methods in control and stabilization includes Wang and Krishnaprasad [1992], Koditschek [1989], Koditschek and Rimon [1990] and Baillieul [1993].

This paper is organized as follows. In $\S 2$, we describe the controlled Lagrangian approach to stabilization. In $\S 3$, we apply the approach to stabilization of an inverted pendulum on a cart. In $\S 4$, we apply the approach to stabilization of rotation of a rigid spacecraft about its unstable middle axis using a single internal rotor. In $\S 5$, we discuss application to the underwater vehicle problem and then give some final remarks.

\section{Controlled Lagrangian Approach}

The controlled Lagrangian approach begins with a mechanical system with an uncontrolled (free) Lagrangian equal to kinetic energy minus potential energy. As mentioned above, we modify the kinetic energy to produce a new controlled Lagrangian which describes the dynamics of the controlled closed-loop system.

Suppose our system has configuration space $Q$ and that, for the purposes of this paper, $Q$ has the form $S \times G$, where $G$ is a Lie group. Our goal will be to control the variables lying in $S$ using controls which act directly on the variables lying in $G$. We assume that the Lagrangian is invariant under the action of $G$ on $Q$, where the action is on the factor $G$ alone. In many specific examples, such as those given below, the invariance is equivalent to the Lagrangian being cyclic in the $G$-variables. Accordingly, this produces a conservation law for the free system. Our construction will preserve the invariance of the Lagrangian, thus providing us with a modified conservation law.

The essence of the modification of the Lagrangian involves changing the metric tensor $g(\cdot, \cdot)$ that defines the kinetic energy $\frac{1}{2} g(\dot{q}, \dot{q})$.

The tangent space to $Q$ can be split into a sum of horizontal and vertical parts defined as follows: for each tangent vector $v_{q}$ to $Q$ at a point $q \in Q$, we can write a unique decomposition

$$
v_{q}=\operatorname{Hor} v_{q}+\operatorname{Ver} v_{q},
$$

such that the vertical part is tangent to $G$ and where the horizontal part is uniquely defined by requiring the identity

$$
g\left(v_{q}, w_{q}\right)=g\left(\operatorname{Hor} v_{q}, \operatorname{Hor} w_{q}\right)+g\left(\operatorname{Ver} v_{q}, \operatorname{Ver} w_{q}\right)
$$

where $v_{q}$ and $w_{q}$ are arbitrary tangent vectors. (This choice of horizontal space coincides with that given by the mechanical connection - see e.g. Marsden [1992].) One can think intuitively of this decomposition of vectors as a decomposition into a piece in the symmetry, or group direction (the vertical piece) and one in the shape, or internal direction (the horizontal piece). For example, in a vibrating molecule, this would correspond to a decomposition into rotational and vibrational modes.

For the kinetic energy of our controlled Lagrangian, we use a modified version of the right hand side of equation (2.1). The potential energy remains unchanged. The modification consists of three ingredients: i) a different choice of horizontal space denoted $\mathrm{Hor}_{\tau}$, ii) a change $g \rightarrow g_{\sigma}$ of the metric acting on horizontal vectors and iii) a change $g \rightarrow g_{\rho}$ of the metric acting on vertical vectors. Corresponding to this change, the controlled Lagrangian takes the form of a modified kinetic energy minus the potential energy:

$$
\begin{aligned}
L_{\tau, \sigma, \rho} & =\frac{1}{2}\left[g_{\sigma}\left(\operatorname{Hor}_{\tau} v_{q}, \operatorname{Hor}_{\tau} w_{q}\right)\right. \\
& \left.+g_{\rho}\left(\operatorname{Ver}_{\tau} v_{q}, \operatorname{Ver}_{\tau} w_{q}\right)\right]-V(q)
\end{aligned}
$$

where $V$ is the potential energy.

The equations corresponding to this Lagrangian are our closed-loop equations. The new terms appearing in those equation corresponding to the directly controlled variables are interpreted as control inputs. The modifications to the Lagrangian are chosen so that no new terms appear in the equations corresponding to the variables that are not directly controlled. We refer to this process as "matching". Further details on the general procedure may be found in Bloch, Marsden and Sánchez de Alvarez [1997] and in a forthcoming paper.

We let $\xi_{Q}$ denote the infinitesimal generator corresponding to a Lie algebra element $\xi$ (see Marsden [1992]). This may be thought of intuitively as infinitesimal group motions of the system. The new horizontal space consists of vectors of the form $\operatorname{Hor}_{\tau} v_{q}=$ Hor $v_{q}-[\tau(v)]_{Q}(q)$ where $\tau$ is a one form that annihilates vertical vectors.

In certain examples, including the inverted pendulum on a cart, we can choose $g_{\rho}=g$ (i.e., there is no $\rho$ needed) and $g_{\sigma}$ to modify the original metric $g$ only in the group directions by a scalar factor $\sigma$. In this case, the controlled Lagrangian takes the form

$$
L_{\tau, \sigma}(v)=L\left(v+[\tau(v)]_{Q}(q)\right)+\frac{\sigma}{2} g\left([\tau(v)]_{Q},[\tau(v)]_{Q}\right) .
$$

For the satellite with rotors, one must include the effects of $\rho$ as well; this modification, consistent with $(2.2)$, is given in (4.3). We remark in passing that the controlled Lagrangian is a modification of the Kaluza-Klein Lagrangian for a particle in a magnetic field, for example (see Marsden and Ratiu [1994]).

The general strategy is: 
1. Start with a mechanical system with a Lagrangian $L$ of the form kinetic minus potential energy and a symmetry group $G$. (In the cart pendulum example below the symmetry group is translation in the horizontal direction).

2. Write down the equations of motion for the uncontrolled system.

3. Introduce $\tau, \sigma$ and $\rho$ to get the controlled Lagrangian (2.2).

4. Write down the equations of motion corresponding to the controlled Lagrangian and read off the control law $u$ from the equations in the symmetry variables (this will be a conservation law).

5. Choose $\tau, \sigma$ and $\rho$ so that the controlled EulerLagrange equations for the original system (i.e., the Euler-Lagrange equations for the Lagrangian $L$ with the control) agree with (match) the Euler-Lagrange equations for the controlled Lagrangian $L_{\tau, \sigma, \rho}$. Determine a feedback law for $u$ by using the Euler-Lagrange equations to eliminate accelerations; then the control law becomes a feedback that is configuration and, possibly, velocity dependent.

6. The stability of an equilibrium is determined by linearization or by the energy-momentum (or energyCasimir-Arnold) method, using any available freedom in the choice of $\tau, \sigma$ and $\rho$.

\section{Inverted Pendulum on a Cart}

We now apply the above ideas to the inverted pendulum on a cart. (The linearized case of this problem was done in Bloch, Marsden and Sánchez de Alvarez [1997].) This example shows the effectiveness of the method for the stabilization of balance systems. Related examples we have in mind are systems like the inverted spherical pendulum on a hockey puck (see conclusions) and the bicycle (see, for example, Getz and Marsden [1994] and Koon and Marsden [1996]).

First, we compute the Lagrangian for the cartpendulum system. Let $s$ denote the position of the cart on the $s$-axis and let $\theta$ denote the angle of the pendulum with the upright vertical, as in the figure.

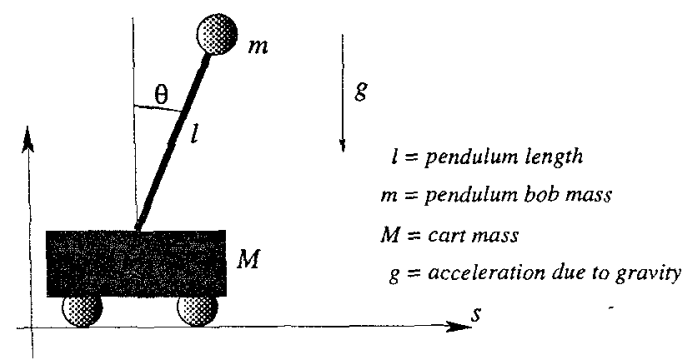

Here, the configuration space is $Q=G \times S=\mathbb{R} \times$ $S^{1}$ with the first factor being the cart position $s$, and the second factor being the pendulum angle, $\theta$. The velocity phase space, $T Q$ has coordinates $(s, \theta, \dot{s}, \dot{\theta})$.
The velocity of the cart relative to the lab frame is $\dot{s}$, while the velocity of the pendulum relative to the lab frame is the vector

$$
v_{\text {pend }}=(\dot{s}+l \cos \theta \dot{\theta},-l \sin \theta \dot{\theta}) .
$$

The system kinetic energy is just the sum of the kinetic energies of the cart and the pendulum:

$$
K\left((s, \theta, \dot{s}, \dot{\theta})=\frac{1}{2}(\dot{s}, \dot{\theta})\left(\begin{array}{cc}
M+m & m l \cos \theta \\
m l \cos \theta & m l^{2}
\end{array}\right)\left(\begin{array}{c}
\dot{s} \\
\dot{\theta}
\end{array}\right) .\right.
$$

The Lagrangian is the kinetic minus potential energy, so we get

$$
L(s, \theta, \dot{s}, \dot{\theta})=K(s, \theta, \dot{s}, \dot{\theta})-V(\theta),
$$

where the potential energy is $V=m g l \cos \theta$.

The symmetry group $G$ of the pendulum-cart system is that of translation in the $s$ variable so $G=\mathbb{R}$. We do not destroy this symmetry when doing stabilization in $\theta$; we would, however, use symmetry breaking potentials to track in the variable $s$ if tracking were our goal. In this paper we are focusing on stabilizing this balance system.

For convenience we rewrite the Lagrangian as

$$
L(s, \theta, \dot{s}, \dot{\theta})=\frac{1}{2}\left(\alpha \dot{\theta}^{2}+2 \beta \cos \theta \dot{s} \dot{\theta}+\gamma \dot{s}^{2}\right)+D \cos \theta,
$$

where $\alpha=m l^{2}, \beta=m l, \gamma=M+m$ and $D=-m g l$ are constants. Note that $\alpha \gamma-\beta^{2}>0$.

The momentum conjugate to $s$ is $p_{s}=\gamma \dot{s}+\beta \cos \theta \dot{\theta}$ and the momentum conjugate to $\theta$ is $p_{\theta}=\alpha \dot{\theta}+\beta \cos \theta \dot{s}$.

The relative equilibrium defined by $\theta=0, \dot{\theta}=0$ and $\dot{s}=0$ is unstable since $D<0$.

The equations of motion of the cart pendulum system with a control force $u$ acting on the cart (and no direct forces acting on the pendulum) are, since $s$ is a cyclic variable,

$$
\begin{gathered}
\frac{d}{d t} \frac{\partial L}{\partial \dot{s}}=u \\
\frac{d}{d t} \frac{\partial L}{\partial \dot{\theta}}-\frac{\partial L}{\partial \theta}=0 \\
\frac{d}{d t} p_{s}=\frac{d}{d t}(\gamma \dot{s}+\beta \cos \theta \dot{\theta})=u \\
\frac{d}{d t} p_{\theta}+\beta \sin \theta \dot{s} \dot{\theta}+D \sin \theta= \\
\frac{d}{d t}(\alpha \dot{\theta}+\beta \cos \theta \dot{s})+\beta \sin \theta \dot{s} \dot{\theta}+D \sin \theta=0(3)
\end{gathered}
$$

Next we form the controlled Lagrangian by modifying only the kinetic energy of the free pendulum cart Lagrangian according to our general formalism. This involves a nontrivial choice of $\tau$ and $\sigma$, but in this case it is sufficient to let $\rho$ be the identity. 
The most general $s$-invariant horizontal one form $\tau$ is given by $\tau=k(\theta) d \theta$ and $\sigma$ is just a scalar. Using (2.3), we let

$$
\begin{aligned}
L_{\tau, \sigma} & :=\frac{1}{2}\left(\alpha \dot{\theta}^{2}+2 \beta \cos \theta(\dot{s}+k \dot{\theta}) \dot{\theta}\right. \\
& \left.+\gamma(\dot{s}+k \dot{\theta})^{2}\right)+\frac{\sigma}{2} \gamma k^{2} \dot{\theta}^{2}+D \cos \theta
\end{aligned}
$$

Notice that the variable $s$ is still cyclic. Following the guidelines of the theory, we look for the feedback control by looking at the change in the conservation law. Associated to the new Lagrangian $L_{\tau, \sigma}$, we have the conservation law

$$
\frac{d}{d t}\left(\frac{\partial L_{\tau, \sigma}}{\partial \dot{s}}\right)=\frac{d}{d t}(\beta \cos \theta \dot{\theta}+\gamma(\dot{s}+k \dot{\theta}))=0,
$$

which we can rewrite in terms of the conjugate momentum $p_{s}$ for the uncontrolled Lagrangian as

$$
\frac{d}{d t} p_{s}=u:=-\frac{d}{d t}(\gamma k(\theta) \dot{\theta}) .
$$

Thus, we identify the term on the right hand side with the control force exerted on the cart.

Still using the controlled Lagrangian, as well as (3.7), the $\theta$ equation is computed to be

$$
\begin{aligned}
& \left(\alpha-\frac{\beta^{2}}{\gamma} \cos ^{2} \theta+\sigma \gamma k^{2}(\theta)\right) \ddot{\theta} \\
& +\left(\frac{\beta^{2}}{\gamma} \cos \theta \sin \theta+\sigma \gamma k(\theta) k^{\prime}(\theta)\right) \dot{\theta}^{2} \\
& +D \sin \theta=0 .
\end{aligned}
$$

For many examples, such as the the rigid body with an internal rotor, it is important here to use the conservation law itself rather than its differentiated form to eliminate the group variables ( $s$ in this case). The resulting system will then depend on the value of the constant chosen for the conservation law.

The next step is to make choices of $k$ and $\sigma$ so that the equation (3.9) using the controlled Lagrangian agrees with the $\theta$ equation for the controlled cart (3.5) with the control law given by equation (3.8). The $\theta$ equation for the controlled cart is

$$
\begin{aligned}
& \left(\alpha-\frac{\beta^{2}}{\gamma} \cos ^{2} \theta-\beta k(\theta) \cos \theta\right) \ddot{\theta} \\
& +\left(\frac{\beta^{2}}{\gamma} \cos \theta \sin \theta+-\beta \cos \theta k^{\prime}(\theta)\right) \dot{\theta}^{2} \\
& +D \sin \theta=0 .
\end{aligned}
$$

Comparing equations (3.9) and (3.10) we see that we require (twice)

$$
\sigma \gamma[k(\theta)]^{2}=-\beta k(\theta) \cos \theta .
$$

Since $\sigma$ was assumed to be a constant we set

$$
k(\theta)=\kappa \cos \theta
$$

where $\kappa$ is a constant (so $\sigma=-\beta / \gamma \kappa$ ).

Then substituting for $\ddot{\theta}$ and $k$ in (3.8) we obtain the desired nonlinear control law:

$$
u=\gamma \kappa\left((\sin \theta) \dot{\theta}^{2}+(\cos \theta) \zeta\right)
$$

where

$$
\zeta=\left[\frac{D \sin \theta+\dot{\theta}^{2}\left(\frac{\beta^{2}}{\gamma}+\beta \kappa\right) \cos \theta \sin \theta}{\alpha-\left(\frac{\beta^{2}}{\gamma}+\beta \kappa\right) \cos ^{2} \theta}\right] .
$$

By examining the linearization of the closed-loop system, one can see that the equilibrium $\theta=\dot{\theta}=\dot{s}=0$ is stable if

$$
\kappa>\frac{\alpha \gamma-\beta^{2}}{\beta \gamma}>0 .
$$

In summary, we get a stabilizing feedback control law for the inverted pendulum provided $\kappa$ satisfies (3.13).

A simple calculation shows the denominator of $\zeta$ is nonzero for $\theta$ satisfying $\sin ^{2} \theta<E / F$ where $E=$ $\kappa-\left(\alpha \gamma-\beta^{2}\right) /(\beta \gamma)$ (which is positive if the stability condition holds) and $F=\kappa+(\beta / \gamma)$. This range of $\theta$ tends to the range $-\pi / 2<\theta<\pi / 2$ for large $\kappa$.

The above remark indicates that the region of stability (or attraction when damping control is added) is the whole range of non-downward pointing states. In fact, we assert that this method produces large computable domains of attraction for stabilization.

This approach has advantages because it is done within the context of mechanics; one can understand the stabilization in terms of the effective creation of an energy well by the feedback control. Note also that the linearized feedback is just proportional feedback.

\section{Rigid Spacecraft with a Symmetric Rotor}

Following Krishnaprasad [1985] and Bloch, Krishnaprasad, Marsden and Sánchez de Alvarez [1992], we consider a rigid body with a rotor aligned along the third principal axis of the body as in the figure. The rotor spins under the influence of a torque $u$ acting on the rotor. The configuration space is $Q=S O(3) \times S^{1}$, with the first factor being the spacecraft attitude and the second factor being the rotor angle. The Lagrangian is total kinetic energy of the system, (rigid carrier plus rotor), with no potential energy.

The Lagrangian for this system is

$$
L=\frac{1}{2}\left(\lambda_{1} \Omega_{1}^{2}+\lambda_{2} \Omega_{2}^{2}+I_{3} \Omega_{3}^{2}+J_{3}\left(\Omega_{3}+\dot{\alpha}\right)^{2}\right)
$$

where $I_{1}>I_{2}>I_{3}$ are the rigid body moments of inertia, $J_{1}=J_{2}$ and $J_{3}$ are the rotor moments of inertia, $\Omega=\left(\Omega_{1}, \Omega_{2}, \Omega_{3}\right)$ is the body angular velocity vector of the carrier and $\alpha$ is the relative angle of the rotor.

The body angular momenta are determined by the Legendre transform to be

$$
\Pi_{1}=\lambda_{1} \Omega_{1}
$$




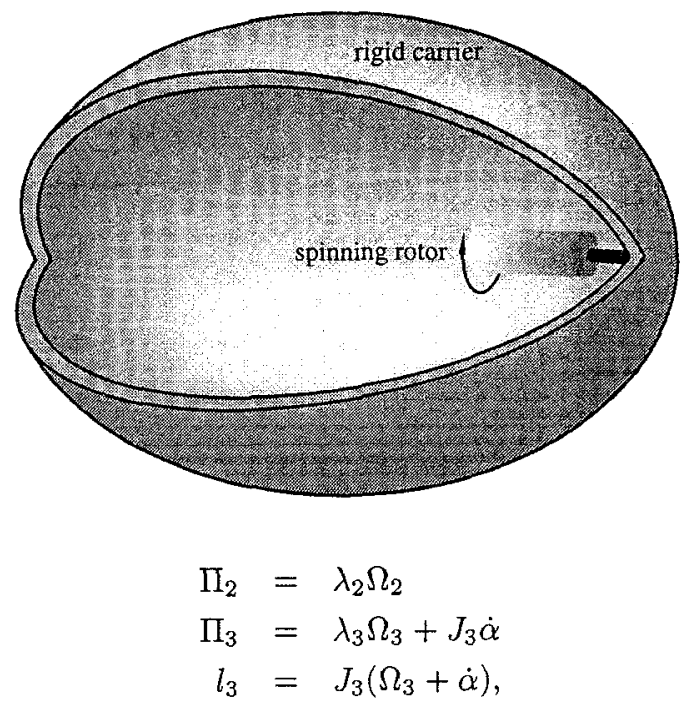

$\lambda_{i}=I_{i}+J_{i}$. The momentum conjugate to $\alpha$ is $l_{3}$.

The equations of motion with a control torque $u$ acting on the rotor are

$$
\begin{aligned}
\lambda_{1} \dot{\Omega}_{1} & =\lambda_{2} \Omega_{2} \Omega_{3}-\left(\lambda_{3} \Omega_{3}+J_{3} \dot{\alpha}\right) \Omega_{2} \\
\lambda_{2} \dot{\Omega}_{2} & =-\lambda_{1} \Omega_{1} \Omega_{3}+\left(\lambda_{3} \Omega_{3}+J_{3} \dot{\alpha}\right) \Omega_{1} \\
\lambda_{3} \dot{\Omega}_{3}+J_{3} \ddot{\alpha} & =\left(\lambda_{1}-\lambda_{2}\right) \Omega_{1} \Omega_{2} \\
\dot{l}_{3} & =u .
\end{aligned}
$$

Now we form the controlled Lagrangian with the group direction being the rotor angle. We choose $\tau$ to be the form $r \Omega_{3}$ where $r$ is a constant scalar. In this case we select the controlled Lagrangian to be

$$
\begin{aligned}
L_{\tau, \sigma, \rho}= & \frac{1}{2}\left(\lambda_{1} \Omega_{1}^{2}+\lambda_{2} \Omega_{1}^{2}+I_{3} \Omega_{3}^{2}\right) \\
& +\frac{1}{2} \rho J_{3}\left((1+r) \Omega_{3}+\dot{\alpha}\right)^{2} \\
& +\frac{1}{2} J_{3} \sigma r^{2} \Omega_{3}^{2} .
\end{aligned}
$$

Notice that this Lagrangian (written in a body frame) depends on all three of $\tau, \rho$ and $\sigma$. To get the control law, we compute the Euler-Lagrange equation associated with $\alpha$. We get

$$
\frac{d}{d t}\left(\frac{\partial L_{\tau, \sigma, \rho}}{\partial \dot{\alpha}}\right)=\frac{d}{d t}\left(\rho J_{3}\left((r+1) \Omega_{3}+\dot{\alpha}\right)\right)=0 .
$$

This can be rewritten in terms of the conjugate momentum $l_{3}$ for the uncontrolled Lagrangian as

$$
\frac{d}{d t} l_{3}=u:=-\frac{d}{d t}\left(J_{3} r \Omega_{3}\right) .
$$

The equations of motion corresponding to $L_{\tau, \sigma, \rho}$ are given as follows. Denoting

$$
\Pi_{3}^{c}=I_{3} \Omega_{3}+\rho J_{3}\left((1+r) \Omega_{3}+\dot{\alpha}\right)(1+r)+J_{3} \sigma r^{2} \Omega_{3},
$$

the remaining equations of motion are

$$
\begin{aligned}
\lambda_{1} \dot{\Omega}_{1} & =\lambda_{2} \Omega_{2} \Omega_{3}-\Pi_{3}^{c} \Omega_{2} \\
\lambda_{2} \dot{\Omega}_{2} & =-\lambda_{1} \Omega_{1} \Omega_{3}+\Pi_{3}^{c} \Omega_{1} \\
\dot{\Pi}_{3}^{c} & =\left(\lambda_{1}-\lambda_{2}\right) \Omega_{1} \Omega_{2} .
\end{aligned}
$$

Matching these to the equations (4.2) with control law defined by (4.5) we find that the systems agree provided one chooses $\sigma=-1 / r$ and $\rho=1 /(1+r)$.

Defining $k$ by

$$
r=\frac{-k}{1-k} \frac{I_{3}}{J_{3}}
$$

and using the equations to eliminate accelerations, one finds the feedback control law defined above is given by

$$
u=k\left(\lambda_{1}-\lambda_{2}\right) \Omega_{1} \Omega_{2} .
$$

Once one has the problem in Lagrangian and hence Hamiltonian form, one can proceed to use the energyCasimir or energy-momentum method to determine stability. This is often much more computationally efficient than an analysis of eigenvalues of the linearized equations (which, in any case, need not imply nonlinear stability in the mechanical case).

As in Bloch, Krishnaprasad, Marsden and Sánchez de Alvarez [1992], we consider the case in which our conserved quantity is equal to zero and the equilibrium is $(0, \bar{M}, 0)$ corresponding to steady rotation about the intermediate axis (unstable for the uncontrolled spacecraft). The energy-Casimir method then shows that

Proposition 4.1 For $k>1-J_{3} / \lambda_{2}$, the equilibrium $(0, \bar{M}, 0)$ is nonlinearly stable for the feedback controlled system.

Indeed, we look at $H+C$ where $C=\varphi\left(\|\Pi\|^{2}\right)$. Pick $\varphi$ so that the first variation vanishes:

$$
\left.\delta(H+C)\right|_{(0, \bar{M}, 0)}=0
$$

One computes that $\delta^{2}(H+C)$ is negative definite if $k>1-J_{3} / \lambda_{2}$ and $\varphi^{\prime \prime}\left(\bar{M}^{2}\right)<0$, which proves the claim.

The stabilization that takes place as the gain is increased can be viewed in terms of a modification of the phase portrait of the rigid body: the four heteroclinic orbits for the rigid body close up along the "hinge" joining the two saddle points forming a circle of fixed points and then open up along a "hinge" joining two stable points, forming a stability island where there were saddle points previously.

One of the advantages of the approach in this paper is that it is systematic, given the class of control Lagrangians we have proposed (of course, we are not excluding the possibility that other interesting classes might be found). For example, with the satellite with rotors one can readily deal with variants of the problem such as putting the rotor along the short axis instead of the long one, or with its axis in some direction other than a principal axis direction.

\section{Stabilization of Underwater Vehicle Dynamics}

The dynamics of an underwater vehicle provides another rich example of the methods of the present paper. 
The techniques proceed somewhat similarly to those for the satellite to show what explicit gains are needed to stabilize an otherwise unstable motion. The underwater vehicle example is much richer, however, because it interacts with the surrounding fluid for both rotational and translational motions, whereas the satellite stabilization problem deals only with the rotational dynamics. Some of the relative equilibria that are of interest are discussed in Leonard [1997a] and Leonard and Marsden [1997].

The bottom line is that stabilization is possible with internal rotors and the techniques introduced here provide explicit gain inequalities similar to those for the satellite. The details of this type of example will be given in a forthcoming publication.

Concluding Remarks. The ideas discussed in this paper can be applied to a fairly large class of higher dimensional mechanical systems with symmetry. An example of this class is the inverted spherical pendulum on a $2 \mathrm{~d}$ cart. We will discuss such systems in a forthcoming publication.

As we have also indicated, we expect that the techniques in this paper can be combined with those of Leonard [1997b] who introduced symmetry-breaking potentials for purposes of stabilizing relative equilibria of underwater vehicles. (The potentials can be for either rotational or translational symmetry breaking). On some level, the two methods can simply be concatenated; if the "balance stability" has been achieved in some of the variables (here the carrier angular velocity variables), then the symmetry breaking potentials can be introduced by additional control forces that do not destroy the achieved stability. On a more detailed level, the problem is not quite as simple as concatenation, because the control forces that achieve balance stability also affect the group directions, but leave some directions free (the geometric phase directions, roughly speaking). For the underwater vehicle, this effect is detailed in Leonard and Marsden [1997]. The present framework should allow one to deal with these issues.

We expect one can extend the techniques for purposes of tracking by forming a tracking function $\mathcal{T}(t)$ by taking the function produced by the energy-Casimir method, but with the relative equilibrium (which is a minimum of the function) replaced by the trajectory one wishes to track. One then computes the total time derivative of $\mathcal{T}(t)$ under the influence of control forces and requires that these control forces decrease $\mathcal{T}(t)$; thus, one is guaranteed to track, perhaps approximately, its minimum. For a relative equilibrium no control forces are needed. This tracking methodology is similar to that of Koditschek and Rimon [1990].

Acknowledgments. We thank John Ballieul, Francesco Bullo, Joel Burdick, Neil Getz, Dan Koditschek, P.S. Krishnaprasad, Richard Murray, Tudor Ratiu and Gloria Sánchez de Alvarez for helpful comments.

\section{References.}

Baillieul, J. [1993] Stable average motions of mechanical systems subject to periodic forcing. Fields Inst. Comm. 1, 1-23.

Bloch, A.M., P.S. Krishnaprasad, J.E. Marsden and G. Sánchez de Alvarez [1992] Stabilization of rigid body dynamics by internal and external torques. Automatica 28, 745-756.

Bloch, A.M., J.E. Marsden, G. Sánchez de Alvarez [1997] Stabilization of relative equilibria of mechanical systems with symmetry. Proc. Notre Dame Conf. on Applied Mathematics, Birkhäuser.

Getz, N.H. and J.E. Marsden [1995] Control for an autonomous bicycle, International Conference on Robotics and Automation, IEEE, Nagoya, Japan.

Koditschek, D.E. [1989] The application of total energy as a Lyapunov function for mechanical control systems, in Dynamics and control of multibody systems (Brunswick, ME, 1988), 131-157, Contemp. Math., 97, Amer. Math. Soc., Providence, RI.

Koditschek, D.E. and E. Rimon [1990] Robot navigation functions on manifolds with boundary. $A d v$. in Appl. Math. 11, 412-442.

Koon, W.S. and J.E. Marsden [1996] The Hamiltonian and Lagrangian approaches to nonholonomic mechanics Caltech preprint.

Krishnaprasad, P.S. [1985] Lie-Poisson structures, dual-spin spacecraft and asymptotic stability, Nonl. Anal. Th. Meth. and Appl. 9, 1011-1035.

Leonard, N.E. [1997a] Stability of a bottom-heavy underwater vehicle, Automatica 33, 331-346.

Leonard, N.E. [1997b] Stabilization of underwater vehicle dynamics with symmetry-breaking potentials, to appear in Systems and Control Letters.

Leonard, N.E. and J.E. Marsden [1997] Stability and drift of underwater vehicle dynamics: Mechanical systems with rigid motion symmetry, Physica $D$ 105, 130-162.

Marsden, J.E. [1992], Lectures on Mechanics London Mathematical Society Lecture note series. 174, Cambridge University Press.

Marsden, J.E. and T.S. Ratiu [1994] Symmetry and Mechanics. Texts in Applied Mathematics, 17, Springer-Verlag.

Wang, L.S. and P.S. Krishnaprasad [1992] Gyroscopic control and stabilization, J. Nonlinear Sci. $\mathbf{2}$, $367-415$ 